\title{
Discovery of CJ-2360 as a Potent and Orally Active Inhibitor of Anaplastic \\ Lymphoma Kinase (ALK) Capable of Achieving Complete Tumor Regression
}

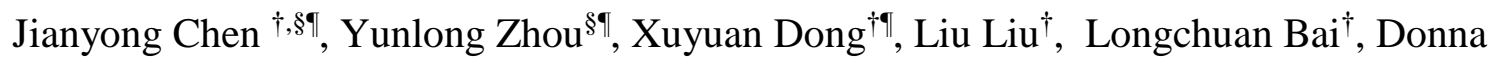
McEachern ${ }^{\dagger}$, Sally Przybranowski ${ }^{\dagger}$, Chao-Yie Yang ${ }^{\dagger}$, Jeanne Stuckey ${ }^{\wedge}$, Xiaoqin $\mathrm{Li}^{\ddagger}$, Bo Wen ${ }^{\ddagger}$,

Ting Zhao ${ }^{\ddagger}$, Siwei Sun ${ }^{\ddagger}$, Duxin Sun ${ }^{\ddagger}$, Lingling Jiao ${ }^{\S}$, Yu Jing ${ }^{\S}$, Ming Guo ${ }^{\S}$, Dajun Yang ${ }^{\S}$, and Shaomeng Wang ${ }^{\dagger *}$

${ }^{\dagger}$ Rogel Cancer Center and Departments of Internal Medicine, Pharmacology, and Medicinal Chemistry, University of Michigan, Ann Arbor, Michigan 48109, United States

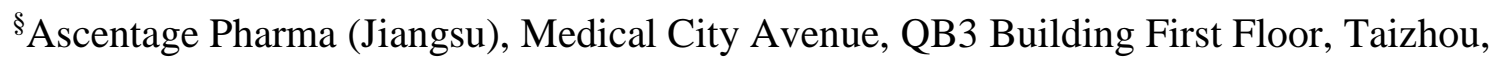
Jiangsu Province 225300 China

${ }^{\Delta}$ Life Sciences Institute, University of Michigan, Ann Arbor, Michigan 48109, United States

tDepartment of Pharmaceutical Sciences, College of Pharmacy, University of Michigan, Ann Arbor, Michigan 48109, United States

IThese authors contribute equally. 
Table S1. Summary of Kinome screening data for CJ-2360 against 468 kinases.

\begin{tabular}{|c|c|c|}
\hline & Name of kinases & $\begin{array}{c}\% \text { of Inhibition by CJ-2360 } \\
\text { at } 100 \mathrm{nM}\end{array}$ \\
\hline 1 & AAK1 & 9 \\
\hline 2 & ABL1(E255K)-phosphorylated & 22 \\
\hline 3 & ABL1(F317I)-nonphosphorylated & 0 \\
\hline 4 & ABL1(F317I)-phosphorylated & 0 \\
\hline 5 & ABL1(F317L)-nonphosphorylated & 0 \\
\hline 6 & ABL1(F317L)-phosphorylated & 2 \\
\hline 7 & ABL1(H396P)-nonphosphorylated & 0 \\
\hline 8 & ABL1(H396P)-phosphorylated & 9 \\
\hline 9 & ABL1(M351T)-phosphorylated & 0 \\
\hline 10 & ABL1(Q252H)-nonphosphorylated & 0 \\
\hline 11 & ABL1(Q252H)-phosphorylated & 0 \\
\hline 12 & ABL1(T315I)-nonphosphorylated & 0 \\
\hline 13 & ABL1(T315I)-phosphorylated & 0 \\
\hline 14 & ABL1(Y253F)-phosphorylated & 0 \\
\hline 15 & ABL1-nonphosphorylated & 10 \\
\hline 16 & ABL1-phosphorylated & 23 \\
\hline 17 & $\mathrm{ABL} 2$ & 10 \\
\hline 18 & ACVR1 & 2 \\
\hline 19 & ACVR1B & 14 \\
\hline 20 & ACVR2A & 6 \\
\hline 21 & ACVR2B & 0 \\
\hline 22 & ACVRL1 & 18 \\
\hline 23 & ADCK3 & 11 \\
\hline 24 & ADCK4 & 1 \\
\hline 25 & AKT1 & 0 \\
\hline 26 & AKT2 & 7 \\
\hline 27 & AKT3 & 2 \\
\hline 28 & ALK & 89 \\
\hline 29 & ALK(C1156Y) & 97.2 \\
\hline 30 & ALK(L1196M) & 86 \\
\hline 31 & AMPK-alpha1 & 10 \\
\hline 32 & AMPK-alpha2 & 1 \\
\hline 33 & ANKK1 & 36 \\
\hline 34 & ARK5 & 1 \\
\hline 35 & ASK1 & 0 \\
\hline 36 & ASK2 & 0 \\
\hline 37 & AURKA & 1 \\
\hline 38 & AURKB & 0 \\
\hline 39 & AURKC & 0 \\
\hline 40 & $\mathrm{AXL}$ & 58 \\
\hline 41 & BIKE & 0 \\
\hline
\end{tabular}




\begin{tabular}{|c|c|c|}
\hline 42 & BLK & 0 \\
\hline 43 & BMPR1A & 21 \\
\hline 44 & BMPR1B & 0 \\
\hline 45 & BMPR2 & 0 \\
\hline 46 & BMX & 7 \\
\hline 47 & BRAF & 0 \\
\hline 48 & BRAF(V600E) & 22 \\
\hline 49 & BRK & 6 \\
\hline 50 & BRSK1 & 0 \\
\hline 51 & BRSK2 & 21 \\
\hline 52 & BTK & 10 \\
\hline 53 & BUB1 & 3 \\
\hline 54 & CAMK1 & 13 \\
\hline 55 & CAMK1B & 2 \\
\hline 56 & CAMK1D & 17 \\
\hline 57 & CAMK1G & 0 \\
\hline 58 & CAMK2A & 15 \\
\hline 59 & CAMK2B & 1 \\
\hline 60 & CAMK2D & 12 \\
\hline 61 & CAMK2G & 12 \\
\hline 62 & CAMK4 & 15 \\
\hline 63 & CAMKK1 & 15 \\
\hline 64 & CAMKK2 & 34 \\
\hline 65 & CASK & 0 \\
\hline 66 & CDC2L1 & 7 \\
\hline 67 & CDC2L2 & 0 \\
\hline 68 & CDC2L5 & 6 \\
\hline 69 & CDK11 & 1 \\
\hline 70 & CDK2 & 8 \\
\hline 71 & CDK3 & 4 \\
\hline 72 & CDK4 & 0 \\
\hline 73 & CDK4-cyclinD1 & 0 \\
\hline 74 & CDK4-cyclinD3 & 0 \\
\hline 75 & CDK5 & 1 \\
\hline 76 & CDK7 & 9 \\
\hline 77 & CDK8 & 9 \\
\hline 78 & CDK9 & 4 \\
\hline 79 & CDKL1 & 23 \\
\hline 80 & CDKL2 & 7 \\
\hline 81 & CDKL3 & 5 \\
\hline 82 & CDKL5 & 0 \\
\hline 83 & CHEK1 & 0 \\
\hline 84 & CHEK2 & 54 \\
\hline 85 & CIT & 20 \\
\hline 86 & CLK1 & 72 \\
\hline
\end{tabular}




\begin{tabular}{|c|c|c|}
\hline 87 & CLK2 & 53 \\
\hline 88 & CLK3 & 18 \\
\hline 89 & CLK4 & 45 \\
\hline 90 & CSF1R & 8 \\
\hline 91 & CSF1R-autoinhibited & 1 \\
\hline 92 & CSK & 10 \\
\hline 93 & CSNK1A1 & 10 \\
\hline 94 & CSNK1A1L & 26 \\
\hline 95 & CSNK1D & 5 \\
\hline 96 & CSNK1E & 25 \\
\hline 97 & CSNK1G1 & 9 \\
\hline 98 & CSNK1G2 & 0 \\
\hline 99 & CSNK1G3 & 21 \\
\hline 100 & CSNK2A1 & 7 \\
\hline 101 & CSNK2A2 & 0 \\
\hline 102 & CTK & 0 \\
\hline 103 & DAPK1 & 71 \\
\hline 104 & DAPK2 & 66 \\
\hline 105 & DAPK3 & 43 \\
\hline 106 & DCAMKL1 & 23 \\
\hline 107 & DCAMKL2 & 6 \\
\hline 108 & DCAMKL3 & 8 \\
\hline 109 & DDR1 & 0 \\
\hline 110 & DDR2 & 2 \\
\hline 111 & DLK & 0 \\
\hline 112 & DMPK & 1 \\
\hline 113 & DMPK2 & 0 \\
\hline 114 & DRAK1 & 1 \\
\hline 115 & DRAK2 & 4 \\
\hline 116 & DYRK1A & 0 \\
\hline 117 & DYRK1B & 0 \\
\hline 118 & DYRK2 & 0 \\
\hline 119 & EGFR & 0 \\
\hline 120 & EGFR(E746-A750del) & 22 \\
\hline 121 & EGFR(G719C) & 11 \\
\hline 122 & EGFR(G719S) & 26 \\
\hline 123 & EGFR(L747-E749del, A750P) & 20 \\
\hline 124 & EGFR(L747-S752del, P753S) & 19 \\
\hline 125 & EGFR(L747-T751 del,Sins) & 0 \\
\hline 126 & EGFR(L858R) & 1 \\
\hline 127 & EGFR(L858R,T790M) & 0 \\
\hline 128 & EGFR(L861Q) & 0 \\
\hline 129 & EGFR(S752-1759del) & 0 \\
\hline 130 & EGFR(T790M) & 7 \\
\hline 131 & EIF2AK1 & 0 \\
\hline
\end{tabular}




\begin{tabular}{|c|c|c|}
\hline 132 & EPHA1 & 0 \\
\hline 133 & EPHA2 & 0 \\
\hline 134 & EPHA3 & 0 \\
\hline 135 & EPHA4 & 4 \\
\hline 136 & EPHA5 & 0 \\
\hline 137 & EPHA6 & 2 \\
\hline 138 & EPHA7 & 0 \\
\hline 139 & EPHA8 & 0 \\
\hline 140 & EPHB1 & 11 \\
\hline 141 & EPHB2 & 4 \\
\hline 142 & EPHB3 & 11 \\
\hline 143 & EPHB4 & 2 \\
\hline 144 & EPHB6 & 13 \\
\hline 145 & ERBB2 & 5 \\
\hline 146 & ERBB3 & 0 \\
\hline 147 & ERBB4 & 21 \\
\hline 148 & ERK1 & 0 \\
\hline 149 & ERK2 & 0 \\
\hline 150 & ERK3 & 0 \\
\hline 151 & ERK4 & 0 \\
\hline 152 & ERK5 & 10 \\
\hline 153 & ERK8 & 20 \\
\hline 154 & ERN1 & 0 \\
\hline 155 & FAK & 6 \\
\hline 156 & FER & 26 \\
\hline 157 & FES & 0 \\
\hline 158 & FGFR1 & 9 \\
\hline 159 & FGFR2 & 0 \\
\hline 160 & FGFR3 & 0 \\
\hline 161 & FGFR3(G697C) & 16 \\
\hline 162 & FGFR4 & 2 \\
\hline 163 & FGR & 20 \\
\hline 164 & FLT1 & 13 \\
\hline 165 & FLT3 & 4 \\
\hline 166 & FLT3(D835H) & 15 \\
\hline 167 & FLT3(D835V) & 62 \\
\hline 168 & FLT3(D835Y) 8176 & 19 \\
\hline 169 & FLT3(ITD) & 31 \\
\hline 170 & FLT3(ITD,D835V) & 61 \\
\hline 171 & FLT3(ITD,F691L) & 7 \\
\hline 172 & FLT3(K663Q) & 4 \\
\hline 173 & FLT3(N841I) & 3 \\
\hline 174 & FLT3(R834Q) & 0 \\
\hline 175 & FLT3-autoinhibited & 0 \\
\hline 176 & FLT4 & 5 \\
\hline
\end{tabular}




\begin{tabular}{|c|c|c|}
\hline 177 & FRK & 4 \\
\hline 178 & FYN & 0 \\
\hline 179 & GAK & 59 \\
\hline 180 & GCN2(Kin.Dom.2,S808G) & 7 \\
\hline 181 & GRK1 & 11 \\
\hline 182 & GRK2 & 1 \\
\hline 183 & GRK3 & 0 \\
\hline 184 & GRK4 & 25 \\
\hline 185 & GRK7 & 3 \\
\hline 186 & GSK3A & 11 \\
\hline 187 & GSK3B & 0 \\
\hline 188 & HASPIN & 0 \\
\hline 189 & $\mathrm{HCK}$ & 23 \\
\hline 190 & HIPK1 & 27 \\
\hline 191 & HIPK2 & 8 \\
\hline 192 & HIPK3 & 0 \\
\hline 193 & HIPK4 & 52 \\
\hline 194 & HPK1 & 3 \\
\hline 195 & HUNK & 0 \\
\hline 196 & ICK & 0 \\
\hline 197 & IGF1R & 0 \\
\hline 198 & IKK-alpha & 2 \\
\hline 199 & IKK-beta & 0 \\
\hline 200 & IKK-epsilon & 0 \\
\hline 201 & INSR & 9 \\
\hline 202 & INSRR & 0 \\
\hline 203 & IRAK1 & 45 \\
\hline 204 & IRAK3 & 0 \\
\hline 205 & IRAK4 & 8 \\
\hline 206 & ITK & 15 \\
\hline 207 & JAK1(JH1domain-catalytic) & 0 \\
\hline 208 & JAK1(JH2domain-pseudokinase) & 6 \\
\hline 209 & JAK2(JH1domain-catalytic) & 12 \\
\hline 210 & JAK3(JH1domain-catalytic) & 1 \\
\hline 211 & JNK1 & 13 \\
\hline 212 & JNK2 & 0 \\
\hline 213 & JNK3 & 0 \\
\hline 214 & KIT & 2 \\
\hline 215 & KIT(A829P) & 0 \\
\hline 216 & $\mathrm{KIT}(\mathrm{D} 816 \mathrm{H})$ & 0 \\
\hline 217 & $\mathrm{KIT}(\mathrm{D} 816 \mathrm{~V})$ & 4 \\
\hline 218 & KIT(L576P) & 12 \\
\hline 219 & KIT(V559D) & 8 \\
\hline 220 & KIT(V559D,T670I) & 7 \\
\hline 221 & KIT(V559D,V654A) & 0 \\
\hline
\end{tabular}




\begin{tabular}{|c|c|c|}
\hline 222 & KIT-autoinhibited & 0 \\
\hline 223 & LATS1 & 0 \\
\hline 224 & LATS2 & 7 \\
\hline 225 & LCK & 24 \\
\hline 226 & LIMK1 & 0 \\
\hline 227 & LIMK2 & 0 \\
\hline 228 & LKB1 & 0 \\
\hline 229 & LOK & 39 \\
\hline 230 & LRRK2 & 11 \\
\hline 231 & LRRK2(G2019S) & 8 \\
\hline 232 & LTK & 92.2 \\
\hline 233 & LYN & 0 \\
\hline 234 & LZK & 0 \\
\hline 235 & MAK & 15 \\
\hline 236 & MAP3K1 & 0 \\
\hline 237 & MAP3K15 & 5 \\
\hline 238 & MAP3K2 & 0 \\
\hline 239 & MAP3K3 & 11 \\
\hline 240 & MAP3K4 & 0 \\
\hline 241 & MAP4K2 & 0 \\
\hline 242 & MAP4K3 & 0 \\
\hline 243 & MAP4K4 & 0 \\
\hline 244 & MAP4K5 & 2 \\
\hline 245 & MAPKAPK2 & 18 \\
\hline 246 & MAPKAPK5 & 0 \\
\hline 247 & MARK1 & 7 \\
\hline 248 & MARK2 & 14 \\
\hline 249 & MARK3 & 27 \\
\hline 250 & MARK4 & 3 \\
\hline 251 & MAST1 & 28 \\
\hline 252 & MEK1 & 0 \\
\hline 253 & MEK2 & 14 \\
\hline 254 & MEK3 & 0 \\
\hline 255 & MEK4 & 0 \\
\hline 256 & MEK5 & 16 \\
\hline 257 & MEK6 & 21 \\
\hline 258 & MELK & 7 \\
\hline 259 & MERTK & 73 \\
\hline 260 & MET & 0 \\
\hline 261 & MET(M1250T) & 0 \\
\hline 262 & $\mathrm{MET}(\mathrm{Y} 1235 \mathrm{D})$ & 35 \\
\hline 263 & MINK & 9 \\
\hline 264 & MKK7 & 0 \\
\hline 265 & MKNK1 & 0 \\
\hline 266 & MKNK2 & 0 \\
\hline
\end{tabular}




\begin{tabular}{|c|c|c|}
\hline 267 & MLCK & 5 \\
\hline 268 & MLK1 & 1 \\
\hline 269 & MLK2 & 17 \\
\hline 270 & MLK3 & 29 \\
\hline 271 & MRCKA & 7 \\
\hline 272 & MRCKB & 0 \\
\hline 273 & MST1 & 0 \\
\hline 274 & MST1R & 6 \\
\hline 275 & MST2 & 0 \\
\hline 276 & MST3 & 11 \\
\hline 277 & MST4 & 0 \\
\hline 278 & MTOR & 1 \\
\hline 279 & MUSK & 0 \\
\hline 280 & MYLK & 43 \\
\hline 281 & MYLK2 & 26 \\
\hline 282 & MYLK4 & 8 \\
\hline 283 & MYO3A & 6 \\
\hline 284 & MYO3B & 0 \\
\hline 285 & NDR1 & 24 \\
\hline 286 & NDR2 & 0 \\
\hline 287 & NEK1 & 0 \\
\hline 288 & NEK10 & 6 \\
\hline 289 & NEK11 & 0 \\
\hline 290 & NEK2 & 0 \\
\hline 291 & NEK3 & 16 \\
\hline 292 & NEK4 & 3 \\
\hline 293 & NEK5 & 5 \\
\hline 294 & NEK6 & 0 \\
\hline 295 & NEK7 & 33 \\
\hline 296 & NEK9 & 10 \\
\hline 297 & NIK & 6 \\
\hline 298 & NIM1 & 0 \\
\hline 299 & NLK & 0 \\
\hline 300 & OSR1 & 0 \\
\hline 301 & p38-alpha & 2 \\
\hline 302 & p38-beta & 10 \\
\hline 303 & p38-delta & 17 \\
\hline 304 & p38-gamma & 30 \\
\hline 305 & PAK1 & 0 \\
\hline 306 & PAK2 & 0 \\
\hline 307 & PAK3 & 24 \\
\hline 308 & PAK4 & 0 \\
\hline 309 & PAK6 & 3 \\
\hline 310 & PAK7 & 0 \\
\hline 311 & PCTK1 & 0 \\
\hline
\end{tabular}




\begin{tabular}{|c|c|c|}
\hline 312 & PCTK2 & 16 \\
\hline 313 & PCTK3 & 11 \\
\hline 314 & PDGFRA & 0 \\
\hline 315 & PDGFRB & 9 \\
\hline 316 & PDPK1 & 3 \\
\hline 317 & PFCDPK1(P.falciparum) & 6 \\
\hline 318 & PFPK5(P.falciparum) & 0 \\
\hline 319 & PFTAIRE2 & 12 \\
\hline 320 & PFTK1 & 21 \\
\hline 321 & PHKG1 & 30 \\
\hline 322 & PHKG2 & 36 \\
\hline 323 & PIK3C2B & 0 \\
\hline 324 & PIK3C2G & 13 \\
\hline 325 & PIK3CA & 7 \\
\hline 326 & PIK3CA(C420R) & 0 \\
\hline 327 & PIK3CA(E542K) & 2 \\
\hline 328 & PIK3CA(E545A) & 19 \\
\hline 329 & PIK3CA(E545K) & 4 \\
\hline 330 & PIK3CA(H1047L) & 0 \\
\hline 331 & PIK3CA(H1047Y) & 3 \\
\hline 332 & PIK3CA(I800L) & 6 \\
\hline 333 & PIK3CA(M1043I) & 0 \\
\hline 334 & PIK3CA(Q546K) & 0 \\
\hline 335 & PIK3CB & 0 \\
\hline 336 & PIK3CD & 0 \\
\hline 337 & PIK3CG & 0 \\
\hline 338 & PIK4CB & 11 \\
\hline 339 & PIKFYVE & 3 \\
\hline 340 & PIM1 & 28 \\
\hline 341 & PIM2 & 50 \\
\hline 342 & PIM3 & 50 \\
\hline 343 & PIP5K1A & 0 \\
\hline 344 & PIP5K1C & 64 \\
\hline 345 & PIP5K2B & 0 \\
\hline 346 & PIP5K2C & 4 \\
\hline 347 & PKAC-alpha & 0 \\
\hline 348 & PKAC-beta & 4 \\
\hline 349 & PKMYT1 & 15 \\
\hline 350 & PKN1 & 25 \\
\hline 351 & PKN2 & 5 \\
\hline 352 & PKNB(M.tuberculosis) & 1 \\
\hline 353 & PLK1 & 0 \\
\hline 354 & PLK2 & 0 \\
\hline 355 & PLK3 & 5 \\
\hline 356 & PLK4 & 0 \\
\hline
\end{tabular}




\begin{tabular}{|c|c|c|}
\hline 357 & PRKCD & 16 \\
\hline 358 & PRKCE & 2 \\
\hline 359 & PRKCH & 9 \\
\hline 360 & PRKCl & 6 \\
\hline 361 & PRKCQ & 0 \\
\hline 362 & PRKD1 & 42 \\
\hline 363 & PRKD2 & 13 \\
\hline 364 & PRKD3 & 0 \\
\hline 365 & PRKG1 & 14 \\
\hline 366 & PRKG2 & 3 \\
\hline 367 & PRKR & 0 \\
\hline 368 & PRKX & 1 \\
\hline 369 & PRP4 & 0 \\
\hline 370 & PYK2 & 23 \\
\hline 371 & QSK & 3 \\
\hline 372 & RAF1 & 20 \\
\hline 373 & RET & 56 \\
\hline 374 & RET(M918T) & 76 \\
\hline 375 & RET(V804L) & 38 \\
\hline 376 & RET(V804M) & 20 \\
\hline 377 & RIOK1 & 37 \\
\hline 378 & RIOK2 & 0 \\
\hline 379 & RIOK3 & 25 \\
\hline 380 & RIPK1 & 0 \\
\hline 381 & RIPK2 & 2 \\
\hline 382 & RIPK4 & 1 \\
\hline 383 & RIPK5 & 0 \\
\hline 384 & ROCK1 & 0 \\
\hline 385 & ROCK2 & 0 \\
\hline 386 & ROS1 & 9 \\
\hline 387 & RPS6KA4(Kin.Dom.1-N-terminal) & 7 \\
\hline 388 & RPS6KA4(Kin.Dom.2-C-terminal) & 33 \\
\hline 389 & RPS6KA5(Kin.Dom.1-N-terminal) & 0 \\
\hline 390 & RPS6KA5(Kin.Dom.2-C-terminal) & 0 \\
\hline 391 & RSK1(Kin.Dom.1-N-terminal) & 18 \\
\hline 392 & RSK1(Kin.Dom.2-C-terminal) & 57 \\
\hline 393 & RSK2(Kin.Dom.1-N-terminal) 95100 & 5 \\
\hline 394 & RSK2(Kin.Dom.2-C-terminal) & 61 \\
\hline 395 & RSK3(Kin.Dom.1-N-terminal) & 1 \\
\hline 396 & RSK3(Kin.Dom.2-C-terminal) & 54 \\
\hline 397 & RSK4(Kin.Dom.1-N-terminal) & 0 \\
\hline 398 & RSK4(Kin.Dom.2-C-terminal) & 53 \\
\hline 399 & S6K1 & 5 \\
\hline 400 & SBK1 & 0 \\
\hline 401 & SGK & 20 \\
\hline
\end{tabular}




\begin{tabular}{|c|c|c|}
\hline 402 & SgK110 & 0 \\
\hline 403 & SGK2 & 5 \\
\hline 404 & SGK3 & 8 \\
\hline 405 & SIK & 1 \\
\hline 406 & SIK2 & 0 \\
\hline 407 & SLK 5991 & 41 \\
\hline 408 & SNARK & 7 \\
\hline 409 & SNRK & 0 \\
\hline 410 & SRC & 0 \\
\hline 411 & SRMS & 12 \\
\hline 412 & SRPK1 & 11 \\
\hline 413 & SRPK2 & 17 \\
\hline 414 & SRPK3 & 19 \\
\hline 415 & STK16 & 9 \\
\hline 416 & STK33 & 60 \\
\hline 417 & STK35 & 0 \\
\hline 418 & STK36 & 6 \\
\hline 419 & STK39 & 8 \\
\hline 420 & SYK & 22 \\
\hline 421 & TAK1 & 3 \\
\hline 422 & TAOK1 & 0 \\
\hline 423 & TAOK2 & 41 \\
\hline 424 & TAOK3 & 0 \\
\hline 425 & TBK1 & 22 \\
\hline 426 & TEC & 14 \\
\hline 427 & TESK1 & 9 \\
\hline 428 & TGFBR1 & 16 \\
\hline 429 & TGFBR2 & 5 \\
\hline 430 & TIE1 & 0 \\
\hline 431 & TIE2 & 19 \\
\hline 432 & TLK1 & 5 \\
\hline 433 & TLK2 & 7 \\
\hline 434 & TNIK & 16 \\
\hline 435 & TNK1 & 9 \\
\hline 436 & TNK2 & 0 \\
\hline 437 & TNNI3K & 0 \\
\hline 438 & TRKA & 0 \\
\hline 439 & TRKB & 0 \\
\hline 440 & TRKC & 0 \\
\hline 441 & TRPM6 & 8 \\
\hline 442 & TSSK1B & 3 \\
\hline 443 & TSSK3 & 0 \\
\hline 444 & TTK & 27 \\
\hline 445 & TXK & 8 \\
\hline 446 & TYK2(JH1domain-catalytic) & 17 \\
\hline
\end{tabular}




\begin{tabular}{|c|c|c|}
\hline 447 & TYK2(JH2domain-pseudokinase) & 9 \\
\hline 448 & TYRO3 & 0 \\
\hline 449 & ULK1 & 0 \\
\hline 450 & ULK2 & 6 \\
\hline 451 & ULK3 & 0 \\
\hline 452 & VEGFR2 & 6 \\
\hline 453 & VPS34 & 24 \\
\hline 454 & VRK2 & 0 \\
\hline 455 & WEE1 & 6 \\
\hline 456 & WEE2 & 26 \\
\hline 457 & WNK1 & 2 \\
\hline 458 & WNK2 & 11 \\
\hline 459 & WNK3 & 7 \\
\hline 460 & WNK4 & 3 \\
\hline 461 & YANK1 & 0 \\
\hline 462 & YANK2 & 13 \\
\hline 463 & YANK3 & 4 \\
\hline 464 & YES & 6 \\
\hline 465 & YSK1 & 0 \\
\hline 466 & YSK4 & 10 \\
\hline 467 & ZAK & 3 \\
\hline 468 & ZAP70 & 0 \\
\hline
\end{tabular}


Figure S1. HPLC trace of CJ-2360.

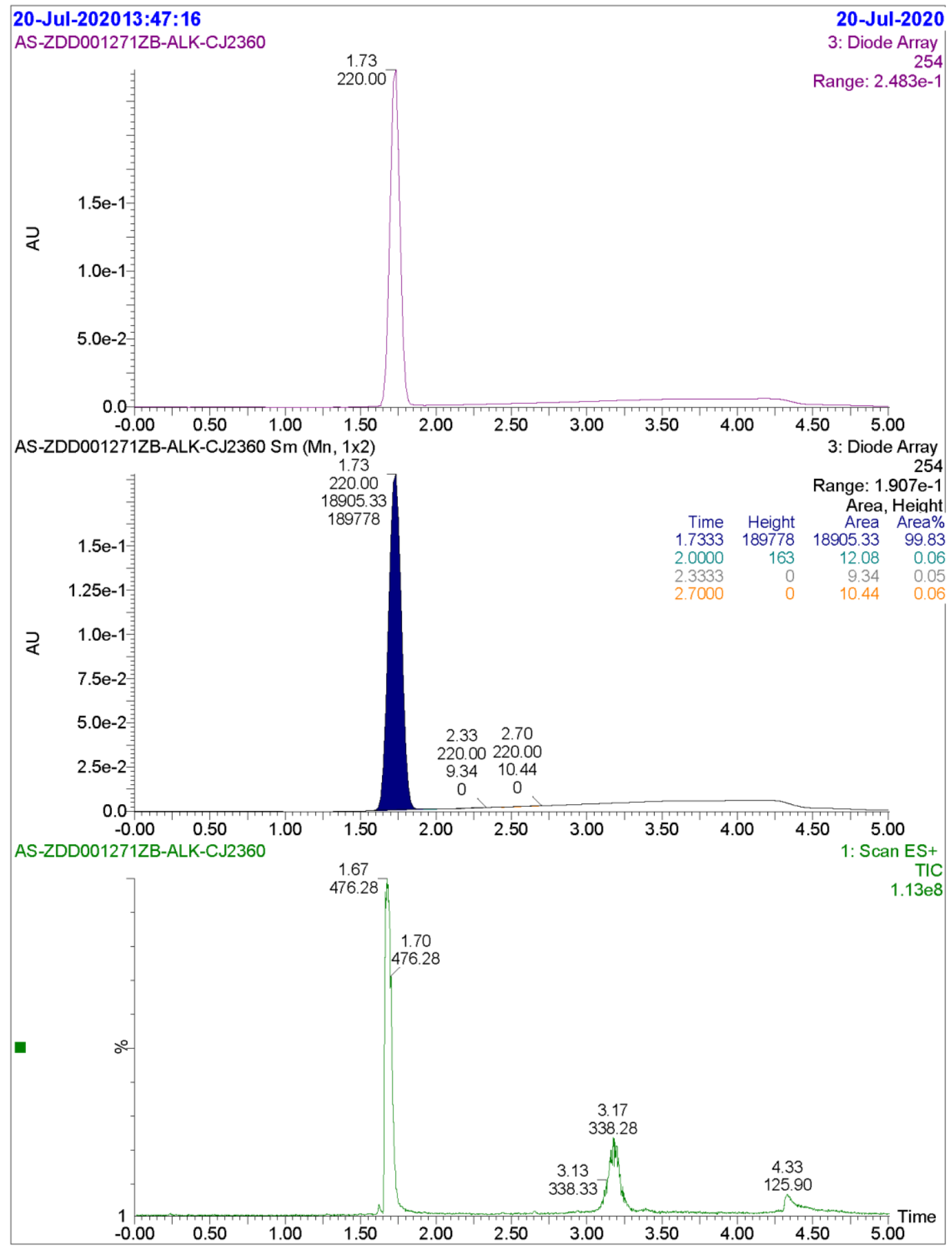

